INTERNATIONAL JOURNAL OF RESEARCHES IN BIOSCIENCES, AGRICULTURE AND TECHNOLOGY (C) VISHWASHANTI MULTIPURPOSE SOCIETY (Global Peace Multipurpose Society) R. No. MH-659/13(N) www.vmsindia.org

\title{
REVIEW ON POLYMER NANOTECHNOLOGY: NANOCOMPOSITES
}

\author{
Y. S. Bopche1, S. R. Bisen ${ }^{2}$ \\ ${ }^{1}$ Dhote Bandhu Science College, Gondia \\ ${ }^{2}$ C. J. Patel College, Tirora \\ yadavbopche@gmail.com
}

\begin{abstract}
:
The present paper describes recent advances in polymer nanocomposites with the primary focus on their advances from basic science to technology. In the large field of nanotechnology, polymer matrix based nanocomposites have become a prominent area of current research and development. Exfoliated clay-based nanocomposites and polymer layered silicate nanocomposites (PLSNCs) have dominated the polymer literature. This review will detail the technology involved with exfoliated clay-based nanocomposites and also include other important areas including barrier properties, flammability resistance, biomedical applications, electrical / electronic / optoelectronic applications and fuel cell benefit. The blend of polymer nanocomposites is an integral aspect of polymer nanotechnology. By inserting the nanometric inorganic compounds, the properties of polymers improve and hence this has a lot of applications depending upon the inorganic material present in the polymers. Solvent casting is one of the easiest and less time consuming methods for the synthesis of polymer nanocomposites. In this paper we present different types of polymer composites, methods of synthesis, characterization techniques and different applications of polymer composites.
\end{abstract}

Keywords: Nanocomposite, nanotechnology, Exfoliated clay, Polymer layered silicates (PLSNCs)

\section{Introduction:}

Field of nanotechnology is one of the most accepted areas for current research and development in all technical discipline including polymer science and technology which covers a broad range of topics - microelectronics or nanoelectronics, polymer-based biomaterials, nanoparticle drug delivery, mini emulsion particles, fuel cell electrode polymer bound catalysts, layer-by-layer self-assembled polymer films, electrospun nanofibers, imprint lithography, polymer blends and nanocomposites. Polymer nanocomposites are materials in which nanoscopic inorganic particles are dispersed in an organic polymer matrix in order to significantly improve the performance properties of the polymers. Polymer nanocomposites represent a new substitute to conventionally filled polymers, because of their nanometer sizes. Filler dispersion nanocomposites exhibit markedly improved properties when compared to pure polymers or their traditional composites or blends which include increased modulus and strength, outstanding barrier properties, improved solvent and heat resistance and decreased flammability. Nanocomposite materials are endowed with many important properties such as nonlinear optical properties, electronic conductivity and luminescence, and have been proposed for their use in various applications including chemical sensors, electroluminescent devices, electro catalysis, batteries, smart windows and memory devices. Layered silicate polymer nanocomposites exhibit superior mechanical characteristics (e.g. $40 \%$ increase of room temperature tensile strength), heat resistance (e.g. $100 \%$ increase in the heat distortion temperature) and chemical resistance (e.g. 10 fold decrease in 02 and $\mathrm{H} 20$ permeability) compared to the neat or traditionally filled resins.[3] These property improvements result from only a 0.1-10 vol. \% addition of the dispersed nanophase. Polyimide-clay hybrids represent another example of polymer nanocomposites which have been prepared by intercalation of the organoclay with a polyamic acid. The clay polyimide hybrid composite films exhibit greatly improved $\mathrm{CO} 2$ barrier properties at low clay content; less than 8.0 vol. \% clay results in almost a ten-fold decrease in permeability. [3]

\section{Material and Methods:}

In recent years considerable efforts have been devoted to the development of methods for the preparation of composite particles consisting of polymer cores covered with shells of different chemical composition. In several of these powders, particles covered with magnetic materials have been used as beads for gas separation, or as pigments, catalysts, coatings, flocculents, toners, raw materials recovery, drug delivery and anticorrosion protection. Adding nanoscale ceramic powders to commercial products can produce another class of polymer nanocomposites. The addition of reinforcing agents is widely used in the production of commodities (packaging films and tyres). It is expected that the reduction of the added particle size down to nanometric scale could enhance the performance of these materials, even though not to the extent as layer addition. These new materials are aimed at being a substitute for 
more expensive technical parts (gear systems in wood drilling machines, wear resistance materials) and in the production of barrier plastic film for food industry. The coatings of magnetic particles are of special interest because of their important applications viz. technological energy transformation, magnetic recording, magnetic fluids and magnetic refrigeration system. Polymer materials have been filled with several inorganic compounds in order to increase properties like heat resistance, mechanical strength and impact resistance and to decrease other properties like electrical conductivity, dielectric constant thereby increasing the permeability for gases like oxygen and water vapor. Polymer composites containing ferrites are increasingly replacing conventional ceramic magnetic materials because of their mouldability and reduction in cost. They are also potential materials for microwave absorbers, sensors and other aerospace applications. These flexible magnets or rubber ferrite composites are possible by the incorporation of magnetic powders in various elastomer matrices. This modifies the physical properties of the polymer matrix considerably. Solvent casting method is one of the easiest methods for the preparation of polymer nanocomposites. It needs simple equipment and is less time consuming. Characterization: The vigorous development of polymeric science and extensive utilization of polymeric materials in technology has led in recent years to the increased interest in the preparation and characterization of polymer and its composite films. Characterization is an essential part of all investigations dealing with materials. The important aspects of characterization are chemical composition, compositional homogeneity (chemical homogeneity), structure, identification and analysis of defects and impurities influencing the properties of the materials. Thus Characterization describes all those features of composition and structure of a material that would suffice for reproducing the material. The advances made in the last few years in characterization techniques, especially in the structure elucidation, have been stupendous and have opened new vistas in solid state materials. Among the several characterization techniques, X-ray diffraction (XRD), scanning electron micrography (SEM) and infrared (IR) spectroscopy and Thermal analysis are main important techniques.[3] Xray Diffraction: X-ray diffraction has played a central role in identifying and characterizing solids since the early part of this century.
Nature of bonding and the working criteria for distinguishing between short-range and longrange order of crystalline arrangements from the amorphous substances are largely derived from $\mathrm{X}$-ray diffraction and thus it remains as a useful tool to obtain structural information. X-ray diffraction pattern of amorphous polymer will not show any sharp and highly intense peaks whereas the nanocomposites of amorphous polymer show sharp and highly intense peaks. This is due to the development of crystallinity in the amorphous polymer. Highly Intense peaks occur in the pattern due to the presence of inserted gamma iron oxide materials in the rubber matrix. X-ray diffraction has been most commonly used for routine characterization as well as for detailed structural elucidation. In order to obtain detailed structural information, knowledge of X-ray diffraction intensities is also essential, the intensities being related to the structure factor. Infrared Spectroscopy: Infrared spectroscopy is one of the most powerful analytical techniques, which offers the possibility of chemical identification. This technique when coupled with intensity measurements may be used for quantitative analysis. One of the important advantages of infrared spectroscopy over' the other usual methods of structural analysis (X-ray diffraction, electron spin resonance, etc.) is that it provides information about the structure of a molecule quickly, without tiresome evaluation methods. This method can solve many problems in organic chemistry (polymeric materials) and coordination chemistry, and also advantageously complements the results obtained by other methods. This technique is based upon the simple fact that a chemical substance shows marked selective absorption in the infrared region giving rise to dose-packed absorption bands called an IR absorption spectrum, over a wide wavelength range. Various bands will be present in the IR spectrum, which will correspond to the characteristic functional groups and bonds present in a chemical substance. Thus an IR spectrum of a chemical substance is a fingerprint for its identification. IR spectrum of polymer nanocomposite shows the presence of both nanomaterials and polymers (depending upon the polymer chain) at various frequencies. Thermal Analysis: Thermal analysis may be defined as the measurement of physical and chemical properties of materials as a function of temperature. Two main thermal analysis techniques are thermo gravimetric analysis (TGA) which automatically records the change in 
weight of a sample as a function of either temperature or time, and the differential thermal analysis (DTA), which measures the difference in temperature $\Delta \mathrm{T}$, between a sample and an inert reference material as a function of temperature; DTA therefore detects change in heat content. A technique closely related but modified to DTA is differential scanning calorimetry (DSC). In DSC, the equipment is designed to allow a quantitative measure of the enthalpy changes, $(\Delta \mathrm{H})$ that occurs in a sample as a function of either temperature or time. DSC is an analytical tool which helps to understand the thermal behavior of polymer nanocomposites. It helps in finding glass transition temperature (Tg) of polymer and its polymer composites. The increase in $\mathrm{Tg}$ values shows the presence of inorganic materials in the polymer matrix.

\section{Results and Discussion:}

A decade of research has shown that nanostructured materials have the potential to significantly impact growth at every level of the world economy in the twenty-first century. This new class of materials is now being introduced in structural applications, such as gas barrier film, flame retardant product and other load bearing applications of particular interest is recently developed nanocomposites consisting of a Exfoliated clay-based nanocomposites and Polymer layered silicate nanocomposites (PLSNCs) because they often exhibit remarkably improved mechanical and other properties when compared with pure polymer or conventional composites. [2][3] In the area of nanotechnology, polymer matrix based nanocomposites have generated a significant amount of attention in the recent literature. This area emerged with the recognition that exfoliated clays could yield significant mechanical property advantages as a modification of polymeric systems [6-8]. The achieved results were at least initially viewed as unexpected offering improved properties over that expected from continuum mechanics predictions. More recent results have, however, indicated that while the property profile is interesting, the clay-based nanocomposites often obey continuum mechanics predictions. There are situations where nanocomposites can exhibit properties not expected with larger scale particulate reinforcements. It is now wellrecognized that the crystallization rate and degree of crystallinity can be influenced by crystallization in confined spaces. Applications: Polymer composites comprising nanoparticles are often investigated where reinforcement of the polymer matrix is achieved. While the reinforcement aspects are a major part of the nanocomposite investigations reported in the literature, many other variants and property enhancements are under active study and in some cases commercialization. The advantages of nanoscale particle incorporation can lead to amyriad of application possibilities where the analogous larger scale particle incorporation would not yield the sufficient property profile for utilization. These areas include barrier properties, membrane separation, UV screens, flammability resistance, polymer blend compatibilization, electrical conductivity, impact modification, and biomedical applications. Examples of nanoparticle, nanoplatelet and nanofiber incorporation into polymer matrices are listed in Table along with potential utility where properties other than mechanical property reinforcement are relevant. [1] \& [2]

Table[1]: Examples of nanoscale filler incorporated in polymer composites for property enhancement other than reinforcement

\begin{tabular}{|l|l|l|}
\hline \multicolumn{1}{|c|}{ Nanofiller } & \multicolumn{1}{|c|}{ Property enhancement (s) } & Application / utility \\
\hline Exfoliated clay & $\begin{array}{l}\text { Flame resistance, barrier, compatibilizer for } \\
\text { polymer blends }\end{array}$ & Safety and mixer \\
\hline SWCNT; MWCNT & Electrical conductivity, charge transport & $\begin{array}{l}\text { Electrical/electronics/ } \\
\text { optoelectronics }\end{array}$ \\
\hline Nanosilver & Antimicrobial & Neutralization \\
\hline ZnO & UV adsorption & UV screens \\
\hline Silica & Viscosity modification & Paint, adhesives \\
\hline CdSe, CdTe & Charge transport & Photovoltaic cells \\
\hline Graphene & Electrical conductivity, barrier, charge transport & Electrical/electronic \\
\hline POSS & Improved stability, flammability resistance & Sensors, LEDs \\
\hline
\end{tabular}




\section{Conclusion}

Novel polymer nanocomposites can be prepared by varying two parameters; first by optimizing polymer to clay or polymer to layered silicate ratio and second by varying the processing techniques [ 1 \& 3]. An exploration in making the polymer nanocomposites with different clays, surfactants and polymers is a continuing subject of research and interest to both academia and industry. Commercial applications: The most publicized application for polymer nanocomposites was an automotive application by Toyota for a timing belt cover. The utilization of exfoliated clay reinforcement of TPO (thermoplastic polyolefin) came later utilized by General Motors for an exterior step assist for another automotive application. [2] Obviously, there are many more applications involving exfoliated clay reinforcement. One of the initial uses for exfoliated clay in barrier applications involved a $20 \mathrm{~mm}$ coating on the interior of a tennis ball to prevent Depressurization. Sports equipment was one of the initial areas where carbon fiber composites were commercialized. This is also true for carbon nanotubes where the carbon nanotubes reinforce the epoxy matrix of the carbon fiber composite in specialty tennis rackets and hockey sticks. Future prospects: Nanocomposites could achieve a dramatic commercial prominence is in advanced composites. Carbon fiber reinforced composites have a limit on the achievable properties due to the low modulus and strength of the matrix phase. Modification of the matrix phase with carbon nanotubes at the lower scale of dimensions and carbon nanofibers at a higher dimensional scale would allow for significant increases in the modulus and strength contributions of the matrix to the overall composite properties. While this would offer some improvement in unidirectional composites, it could be dramatic in the case of cross-ply composites which are the major type of composite structure utilized in advanced composite applications. This concept is under present consideration and could allow a step change in the advanced composite field[2]. These improvements are key to future aircraft and wind energy turbine applications. This approach is analogous to naturally occurring composite structures where the hierarchical construction method employs several dimensional scales beginning at the nanolevel. The same concept is also relevant to the more commodity reinforced composites where exfoliated clay could be added to the matrix for unsaturated polyester-fiberglass composites or other fiberglass reinforced matrix polymers. Carbon nanotube or exfoliated graphite offers substantial opportunities in the electrical / electronic / optoelectronics areas as well as potential in specific emerging technologies. One specific area would be replacement of ITO as a transparent conductor for lower cost and flexible devices. Carbon nanotube sheets have been proposed [5] and the potential for carbon nanotube conjugated polymer composites would be of interest if sufficient electrical conductivity can be obtained $(>103 \mathrm{~S} / \mathrm{cm})$. The potential of low cost graphene production is yet to be realized and large scale utility will be awaiting the synthetic breakthrough. Development of the PLSNCs is one of the latest evolutionary steps of the polymer technology. The PLSNCs offer attractive potential for diversification and application of conventional polymeric materials. Some of PLSNCs are already commercially available and applied in industrial products. Biodegradable polymers based nanocomposites have a great deal of future promise for potential applications as high performance biodegradable materials[1]. These are entirely new types of materials based on plant and nature materials (organoclay). When disposed of in compost, these are safely decomposed into $\mathrm{CO} 2$, water and humus through the activity of microorganisms. The $\mathrm{CO} 2$ and water will become corn or sugarcane again through plant photosynthesis. Undoubtedly, these unique properties originating from the controlled nanostructure pave the way for much broader range of applications and open a new dimension for plastics and composites. At present, it is difficult to predict which, if any, market sector would not be able to benefit from this technology. Therefore, it may well be that polymeric nanocomposites in the mid and long term will pervade all aspects of life, similar to the way plastics did in the last century. Clearly a diverse range of sectors such as aerospace, automotive, packaging (particularly food but also solar cells), electrical and electronic goods, household goods, etc. will profit substantially from a new range of materials which would be offered by this technology.

\section{References:}

1. M. Okamoto; Recent advances in polymer/layered silicate nanocomposites: an overview from science to technology; Page No. 7, Vol. 22, Material Science and Technology. 
2. D.R.Paul, L.M.Robeson; Polymer nanotechnology: Nanocomposites; j.polymer.2008.04.017.

3. Arunkumar Lagashetty, A Venkataraman; Polymer Nanocomposites; Resonance July 2005.

4. Shadpour Mallakpour,Zahra Rafiee; New developments in polymer science and technology using combination of ionic liquids and microwave irradiation; j.progpolymsci.2011.03.001.

5. Zhang M, Fang S, Zakhidov AA, Lee SB,
Aliev AE, Williams CD, et al. Science 2005;309:1215-9.

6. Kojima Y, Usuki A, Kawasumi M, Okada A, Kurauchi T, Kamigaito O. J Polym Sci Part A Polym Chem 1993;31:983-6.

7. Okada A. Fukushim Y, Kawasumi M, Inagaki S, Usuki A, Sugiyama $\mathbf{S}$, et al. US Patent 4,739,007;1988.

8. Kawasumi M. J Polym Sci Part A Polym Chem. 2004; 42:819-24. 9. Y.S.Bopche, D.S.Choudhary, Sonali R.Bisen; Advances in Polymer Nanotechnology: Nanocomposites. 